\title{
LETTERS
}

\section{Dilemmas in the recognition of Sjögren syndrome}

In their CMAJ Practice article, Bau and colleagues present a concise working summary of primary Sjögren syndrome. ${ }^{1}$ Their case definition relied on international criteria that were defined in the 2017 report from the American College of Rheumatology and European League Against Rheumatism. ${ }^{2}$ The latter establishes a set of clinical and investigative findings that create the provisional diagnosis of primary Sjögren syndrome. In essence, the "primary" syndrome would be established as a standalone diagnosis. A "secondary" Sjögren syndrome would then be one in which another disease, most commonly autoimmune, would coexist or perhaps be causal.

A practical clinical problem arises, however, when the clinical presentation may be a forme fruste (atypical or incomplete) of Sjögren syndrome regardless of whether it is deemed primary or secondary. That is, the inclusion and exclusion criteria from the international standard may be present, but the patient does not fully achieve a sufficient scoring from the 5 criteria items. Furthermore, although a patient may be considered to have primary disease when no other coexisting disease is found, the specific diagnosis of another coexisting disease may be in debate or not obvious.

Such dilemmas are illustrated by the findings of "sicca" in the context of autoimmune/ inflammatory syndrome induced by adjuvants (ASIA), which has become an entity of growing interest. ${ }^{3-7}$ A Sjögren syndrome scenario may be in evolution but not fully emerged to the point of fulfilling the current international criteria for Sjögren syndrome. The presence of another associated illness such as ASIA may not be understood or may remain elusive for a period of time. Such patients may be viewed as having an undifferentiated connective tissue disease associated with symptoms of sicca. It is quite likely that many such patients are cared for at a time when a full clinical diagnosis of primary or secondary Sjögren syndrome is not realized.

Clinicians need to maintain an open and investigative mind for these complex patients as they present in more early phases of disease or in confusing contexts.

\section{Nevio Cimolai MD}

Professor, Department of Pathology and Laboratory Medicine, Faculty of Medicine, The University of British Columbia, Vancouver, BC
Cite as: CMAJ 2019 October 7;191:

E1110. doi: 10.1503/cmaj.73159

\section{References}

1. Bau JT, Ivory C, Power B, et al. Multisystem presentation of primary Sjögren syndrome. CMAJ 2019; 191:E446-9.

2. Shiboski CH, Shiboski SC, Seror R, et al. 2016 American College of Rheumatology/European League Against Rheumatism classification criteria for primary Sjögren's Syndrome. Arthritis Rheumatol 2017;69:35-45.

3. Shoaib BO, Patten BM. Human adjuvant disease: presentation as a multiple sclerosis-like syndrome. South Med J 1996;89:179-88.

4. Alijotas-Reig J, Estvere-Valverde E, Gil-Aliberas N, et al. Autoimmune/inflammatory syndrome induced by adjuvants - ASIA - related to biomaterials: analysis of 45 cases and comprehensive review of the literature. Immunol Res 2018;66:120-40.

5. Colaris MJL, de Boer M, van der Hulst RR, et al. Two hundred cases of ASIA syndrome following silicone implants: a comparative study of 30 years and a review of current literature. Immunol Res 2017;65:120-8.

6. Watad A, Quaresma M, Bragazzi NL, et al. The autoimmune/inflammatory syndrome induced by adjuvants (ASIA)/Shoenfeld's syndrome: descriptive analysis of 300 patients from the international ASIA syndrome registry. Clin Rheumatol 2018; 37:483-93.

7. Watad A, Bragazzi NL, McGonagle D, et al. Autoimmune/ inflammatory syndrome induced by adjuvants (ASIA) demonstrates distinct autoimmune and autoinflammatory disease associations according to the adjuvant subtype: insights from an analysis of 500 cases. Clin Immunol 2019;203:1-8.

Competing interests: None declared. 